\title{
Calibration of the Cepheid Distance Scale with the Infrared Surface Brightness Technique
}

\author{
Wolfgang Gieren \\ Universidad de Concepción, Departamento de Física, Astronomy Group, \\ Casilla 4009, Concepción, Chile
}

Pascal Fouqué

European Southern Observatory, Casilla 19001, Santiago 19, Chile; and Observatoire de Paris-Meudon, DESPA, F-92195 Meudon CEDEX, France

\begin{abstract}
.
We review the process of determining the distances to Cepheid variables with the infrared surface brightness technique. We show that both versions of this technique (working with the $V, V-K$ and the pure infrared $K, J-K$ magnitude/color combinations) yield consistent distances to Cepheid variables. For a Cepheid with average-quality photometric and radial velocity data, the typical total uncertainty in the distance is $\pm 5 \%$. We use the distances of 34 Galactic variables to calibrate periodluminosity relations in the $V, I, J, H, K$ bands and demonstrate that these relations lead to distance moduli of the LMC which agree to $\pm 0.02 \mathrm{mag}$. The infrared surface brightness technique yields a best LMC distance modulus of $18.46 \pm 0.02$. We discuss possible metallicity corrections, such as the ones determined by Sasselov et al. from EROS Cepheids comparison in the LMC and SMC, and conclude that our distance modulus is negligibly affected by these corrections. Finally, we present a program to obtain reddening- and metallicity-independent infrared surface brightness distances of Cepheid variables in LMC clusters which will yield an even more accurate mean LMC distance independent of any metallicity correction to Cepheid absolute magnitudes.
\end{abstract}

\section{Introduction}

Cepheid variables continue to be the key primary objects to calibrate the extragalactic distance scale. In view of the results of the HST Key Project on the determination of $\mathrm{H}_{0}$ from Cepheid variables in some 20 nearby galaxies out to the Virgo and Fornax clusters, it is more important than ever to provide a local and accurate calibration of the PL relation (in different bands, particularly in the $V$ and $I$ bands used by HST), and to establish the true distance to the $\mathrm{LMC}$, whose value as derived from a variety of independent methods is still scattering between extremes of 18.1 to 18.7 (see the review of M. Feast in this volume). Also, the size of metallicity corrections to Cepheid absolute mag- 
nitudes is still under dispute (see Kennicutt et al. 1998, and references therein), and it is therefore desirable to have a method which is able to yield direct distances to Cepheid variables, in at least some of the nearest galaxies, which are independent, at least to first order, of the metallicity of the parent galaxy. Recently, such a technique has become available with the introduction of the infrared surface brightness method by Fouqué \& Gieren (1997) which is capable of yielding distances to Cepheid variables nearly independent of adopted absorption corrections and the stellar metallicity, and is therefore an almost ideal tool to determine distances with very low systematic uncertainties to a set of nearby galaxies, most importantly to the LMC. The infrared surface brightness technique is of the Baade-Wesselink type and has smaller systematic uncertainties than its visual counterpart which has been extensively used by Gieren, Barnes \& Moffett (1993) to study Cepheids in the Galaxy.

The use of Baade-Wesselink type methods, with their specific advantages when calibrated and applied in the near-infrared, has gained increased importance since it has been recognized from the HIPPARCOS data that the locations of the main sequences of open clusters can differ by as much as 0.6 mag (Mermilliod et al. 1997; Robichon et al. 1997; van Leeuwen \& Hansen Ruiz 1997), affecting directly the classical approach to calibrate the Cepheid PL relation from the ZAMS-fitting method using Cepheid variables found in open clusters. Also, there is still considerable systematic uncertainty in the LMC distance modulus derived by Feast \& Catchpole (1997) from HIPPARCOS parallaxes of nearby Cepheids, mainly due to the very small signal-to-noise ratios and the subsequent problem of correcting statistical biases, such as the Lutz-Kelker bias (Oudmaijer et al. 1998), and to the fact that a large fraction of Cepheids in the Feast \& Catchpole sample are spectroscopic binaries with orbital periods in the order of the HIPPARCOS observing time interval which may systematically affect the parallaxes of these stars (Szabados 1997).

In this review we concentrate on a description of the infrared surface brightness technique and present its main features and an evaluation of its uncertainties. We then present the results of our calibration of the Galactic Cepheid PL relations from the infrared distances and apply these relations to find the distance to the LMC. Finally, we briefly discuss ongoing programs of applying the technique directly to Cepheids in the Magellanic Clouds. Among recent excellent reviews which examine other issues related to the use of Cepheids as distance indicators, we mention Feast \& Walker (1987), Madore \& Freedman (1991), Welch (in Jacoby et al. 1992), and Tanvir (1997).

\section{The Infrared Surface Brightness (ISB) Technique}

\subsection{Calibration of the Technique}

A detailed description of the technique is given in the paper of Fouque \& Gieren (1997). A visual surface brightness parameter $F_{\mathrm{V}}$ is defined as

$$
F_{\mathrm{V}}=a-0.1 S_{\mathrm{V}}
$$

where $S_{\mathrm{V}}$ is the visual surface brightness defined in terms of the absorptioncorrected apparent magnitude $V_{o}$ and the stellar angular diameter $\Phi$ as 


$$
S_{\mathrm{V}}=V_{\circ}+5 \log \Phi
$$

and the constant $a$ is given by solar quantities as

$$
a=0.1 M_{\odot}^{\text {bol }}+1+0.25 \log \left(4 f_{\odot} / \sigma\right)=4.2207
$$

The heart of the method is to calibrate $F_{\mathrm{V}}$, or a corresponding parameter $F_{\mathrm{K}}$ defined in the $K$ photometric band, in terms of a suitable color index, using interferometrically determined angular diameters of a set of stable giants and supergiants in the color range of Cepheid variables. Once such a calibration is available, Cepheid angular diameters, at any phase of the pulsation cycle, can be found from an absorption-corrected magnitude and color index at this phase. This angular diameter can be combined with the value of the linear displacement from the stellar mean diameter at this same phase which is found from an integration of the observed radial velocity curve of the variable which first has to be transformed to the corresponding pulsational velocity curve by multiplying with a factor (the $p$ factor) which corrects for the effects of averaging the radial velocity over the stellar disk, and limb-darkening. The equation

$$
2\left(R_{\mathrm{o}}+\Delta R\right)=d \Phi
$$

(units: $\Phi$ in arcsec, radius $R$ in $\mathrm{AU}$, distance $d$ in pc) can then be solved for the distance and mean radius of a Cepheid variable using the observed pairs of linear displacements and angular diameters covering the pulsation cycle.

Laney \& Stobie (1995) have demonstrated that the $V-K$ and $J-K$ colors are almost unaffected by the gravity and microturbulence variations of Cepheids during their pulsations and are near-ideal predictors of the surface brightnesses of these variables, as opposed to optical colors like $B-V$ or $V-R$. We therefore decided to calibrate two versions of the ISB technique using the $V, V-K$ and $K, J-K$ magnitude-color combinations. Surface brightness parameters in the $V$ and $K$ bands were calculated for 28 cool giant and supergiant stars from their absorption-corrected magnitudes and measured angular diameters (accurate to better than 5 percent in all cases) which were then correlated with their intrinsic $V-K$ and $J-K$ colors. Excellent linear relations were obtained for both giants and supergiants over a large color range including the one covered by Cepheid variables. There is no systematic difference in these relations between giants and supergiants. The slope of the surface brightness-color relations obtained from the giants/supergiants sample was found to be consistent with the slope obtained from the Cepheids themselves using Thompson's method (Thompson 1975). For our final surface brightness-color relations, we adopted the slopes from the Cepheids and the zero point as coming from the angular diameters of the cool giants and supergiants. This procedure leads to the following relations for the angular diameters (in mas) of a Cepheid in terms of the unreddened magnitudes and colors:

$$
\begin{aligned}
& \Phi=\operatorname{dex}\left(0.5474-0.2 V_{\circ}+0.262(V-K)_{\circ}\right) \\
& \Phi=\operatorname{dex}\left(0.5474-0.2 K_{\circ}+0.220(J-K)_{\circ}\right)
\end{aligned}
$$



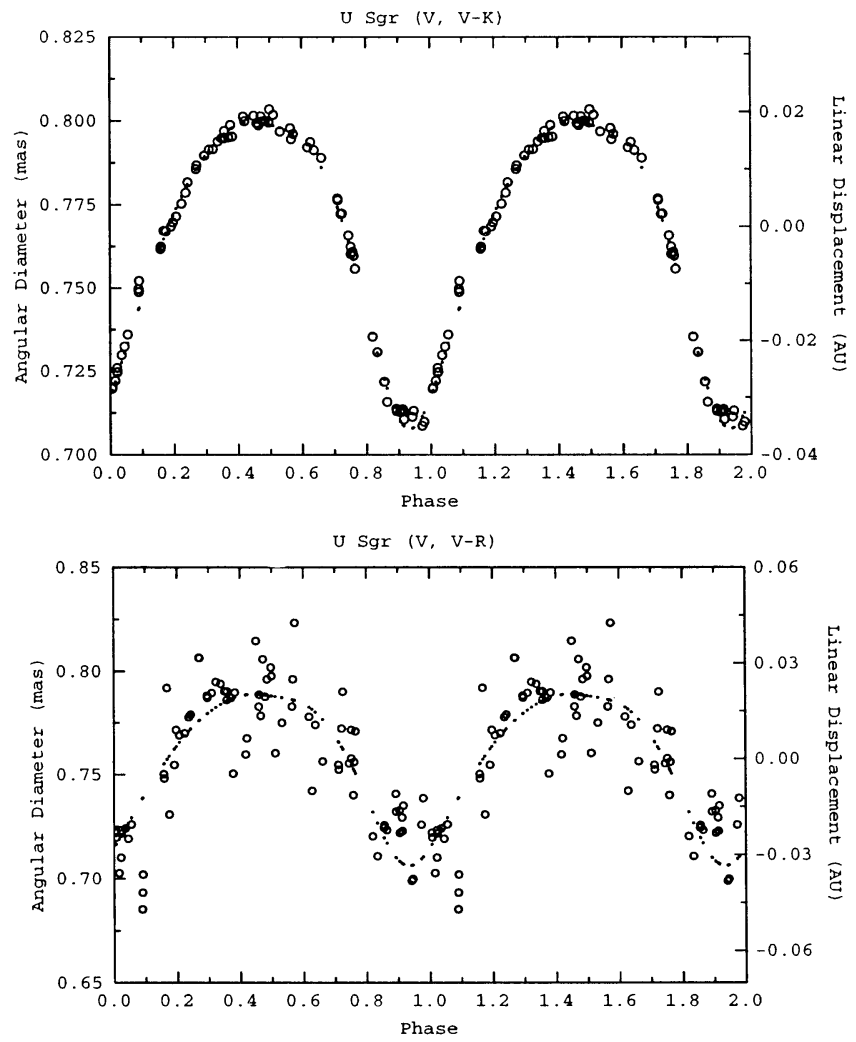

Figure 1. (top) The angular diameters (open circles) of the Cepheid U Sgr calculated from $V, V-K$ photometry according to equation (5) (left scale), plotted against phase. Overplotted (dots) is the linear displacement variation of the Cepheid (right scale). (bottom) The same adjustment from the $V, V-R_{\mathrm{J}}$ photometry.

The angular diameters calculated from these equations are then combined with the linear radius displacements to solve equation (4) for the distance and mean radius of a Cepheid variable. A typical example is shown in Figure 1 where the technique is applied to the Cepheid U Sgr. For comparison, we also plot the angular diameters as obtained from a visual $V, V-R$ surface brightness technique: the dramatic increase in the accuracy of the infrared-based angular diameters over optical ones is evident.

\subsection{Results and Uncertainties}

The main results from an application of both versions of the ISB technique to a sample of 34 Galactic Cepheids with high-quality infrared photometry and radial velocity curves are the following:

- Both techniques produce consistent distances. The mean distance ratio is $0.983 \pm 0.013$. 
- The most important observational constraint to the achievable distance precision is the amplitude of the color curve used in the solution.

- For Cepheids with average-quality data and color curve amplitudes larger than $\sim 0.2 \mathrm{mag}$, the total uncertainty of an infrared distance is $\sim \pm 5$ percent. This uncertainty includes possible systematic errors.

- For Cepheids with very high-quality data, a distance accurate to \pm 3 percent is achievable.

- The ISB distances are practically unaffected by adopted absorption corrections and the metallicity of the Cepheid, a property which makes the technique ideally suited to a direct application to extragalactic Cepheids.

A more detailed account of the effect of a variety of sources of error on the distances can be found in the papers of Gieren, Fouqué \& Gómez $(1997,1998)$.

\section{Period-luminosity Relations in the $V, I, J, H$ and $K$ Bands}

Our approach to calibrate the PL relations is to determine the slopes of the relations, in each band, from samples of LMC Cepheids and to set the zero points from the infrared distances to the Galactic Cepheids. Due to the fact that LMC Cepheids are all at nearly the same distance, it is obvious that the slopes of the PL relations can be obtained with higher accuracy from LMC Cepheids than from Galactic ones. As the most suited LMC Cepheid data sets, we used a sample of 53 Cepheids from Tanvir (1997) for the $V, I$ calibration, supplemented with high-quality data of Moffett et al. (1998), and a sample of 59 Cepheids for the $J, H, K$ calibrations with mean intensity magnitudes and individual reddenings from the SAAO database (Caldwell 1997). We forced the slopes of the LMC Cepheid PL relations to the Galactic Cepheid PL relations and obtained the following absolute calibrations:

$$
\begin{aligned}
M_{\mathrm{V}} & =-2.769( \pm 0.073) \times(\log P-1.0)-4.063( \pm 0.034), \\
M_{\mathrm{I}} & =-3.041( \pm 0.054) \times(\log P-1.0)-4.767( \pm 0.029), \\
M_{\mathrm{J}} & =-3.129( \pm 0.052) \times(\log P-1.0)-5.240( \pm 0.028), \\
M_{\mathrm{H}} & =-3.249( \pm 0.044) \times(\log P-1.0)-5.628( \pm 0.026), \\
M_{\mathrm{K}} & =-3.267( \pm 0.042) \times(\log P-1.0)-5.701( \pm 0.025) .
\end{aligned}
$$

The rms dispersions of these relations range from 0.209 in $V$ to 0.173 in $K$. These are the most accurate PL relations as yet derived from Galactic Cepheids and can be used to derive distances to galaxies of near-solar metallicity. We note that our calibrations yield Cepheid absolute magnitudes which are on average about 0.1 mag fainter than the ones derived by Madore \& Freedman (1991) but are consistent with these within the uncertainties. We also note that the rms dispersion of the $K$ band PL relation (almost unaffected by reddening errors), as compared to the dispersion of the LMC Cepheid $K$ band PL relation $(0.11 \mathrm{mag})$, indicates a maximum error of \pm 6 percent in the ISB distances to the Galactic Cepheids, in agreement with our error budget. 


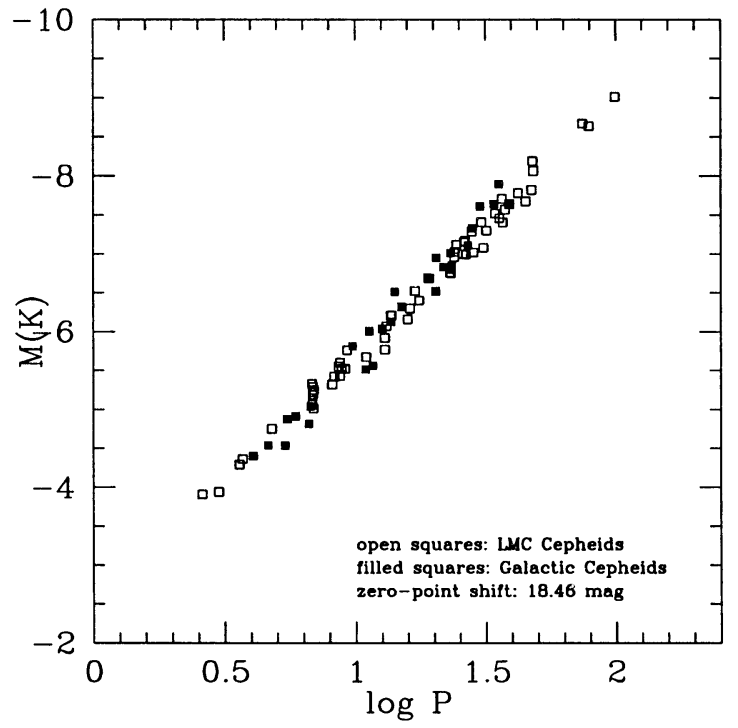

Figure 2. $\quad K$ band PL relations as defined by 59 LMC Cepheids, and by infrared distances of 28 Galactic Cepheids. Best agreement between the data sets occurs for a zero point shift of 18.46 .

\section{The Distance of the LMC}

A comparison of our absolute calibrations of the PL relations to the corresponding relations as defined by the LMC Cepheids yields the distance to the LMC, as the difference of the zero points. An example is shown in Figure 2 for the $K$ band relations. In this way we obtain the LMC distance moduli given in Table 1 from the data in the different bands. It can be appreciated that the agreement of the $\mu_{\mathrm{o}}$ values is excellent, and we obtain a mean LMC true distance modulus of 18.46 with an internal uncertainty as low as $\pm 0.02 \mathrm{mag}$.

Recent work of the HST Key Project team on Cepheids in M 101 yields a metallicity dependence of $\delta(m-M)_{\circ} / \delta[\mathrm{O} / \mathrm{H}]=-0.24 \pm 0.16 \mathrm{mag}$ (Kennicutt et al. 1998) in the $V, I$ spectral range, in the sense that metal-poor Cepheids appear fainter and more distant than more metal-rich stars. However, this correction cannot be directly applied to our $V$ and $I$ distance moduli, because it combines the metallicity effects on magnitude and on color, due to the fact that the HST Key Project calculates Cepheid fields reddenings from the $V$ and $I$ measures themselves. In our case, mean reddenings are adopted from literature, and we are only concerned by the metallicity effect on magnitude. This has been derived by Sasselov et al. (1997) from a comparison of Cepheids in the LMC and the SMC, detected in the EROS survey. They found a magnitude effect of $0.06 \pm 0.01$ in $V$ and $-0.01 \pm 0.01$ in I for the metallicity difference of 0.35 between the LMC and the SMC. As this is approximately the metallicity difference between the LMC and our Galactic Cepheids (Kennicutt et al. 1998 give [O/H] (LMC) = -0.40 and Luck et al. 1998 find $[\mathrm{Fe} / \mathrm{H}](\mathrm{LMC})=-0.3)$, we adopt the Sasselov et al. corrections and derive corrected $V$ and $I$ moduli of $18.42 \pm 0.05$ and 
$18.49 \pm 0.05$, respectively, giving an average $V I$ metallicity-corrected distance modulus of $18.46 \pm 0.04$, in excellent agreement with our adopted value.

An obvious way to circumvent the problem of a metallicity correction is to apply the metallicity-independent ISB method directly to LMC Cepheids. Among the Cepheid variables in the LMC, those in Cepheid-rich LMC clusters as found in the surveys of Welch, Mateo \& Olszewski (1993) and more recently by the MACHO collaboration are obviously the ideal targets. Studies of this kind are underway for the clusters NGC 1866 and 2031 (Gieren, Fouqué \& Storm, 1999 ) and should yield a mean LMC distance accurate to \pm 2 percent which is not affected by the metallicity difference between the LMC and the Galaxy.

Table 1. LMC Cepheid Period-Luminosity Relations and LMC Distance Moduli.

\begin{tabular}{lcccccc}
\hline Band & Slope & $\begin{array}{c}\text { ZP } \\
\log P=1\end{array}$ & rms & $\begin{array}{c}\text { correlation } \\
\text { coefficient }\end{array}$ & $\mathrm{N}$ & $\begin{array}{c}\mu_{\circ} \\
(\mathrm{LMC})\end{array}$ \\
\hline$V$ & -2.769 & 14.397 & 0.204 & 0.983 & 53 & $\mathbf{1 8 . 4 7 8} \pm 0.049$ \\
$I_{\mathrm{C}}$ & -3.041 & 13.693 & 0.150 & 0.992 & 53 & $\mathbf{1 8 . 4 8 2} \pm 0.044$ \\
$J$ & -3.129 & 13.220 & $\mathbf{0 . 1 4 6}$ & 0.992 & 59 & $\mathbf{1 8 . 4 3 3} \pm 0.039$ \\
$H$ & -3.249 & 12.832 & $\mathbf{0 . 1 2 4}$ & 0.995 & 59 & $\mathbf{1 8 . 4 4 3} \pm 0.039$ \\
$K$ & -3.267 & $\mathbf{1 2 . 7 5 9}$ & $\mathbf{0 . 1 1 8}$ & 0.995 & $\mathbf{5 9}$ & $\mathbf{1 8 . 4 5 7 \pm 0 . 0 3 9}$ \\
\hline \hline
\end{tabular}

Acknowledgments. This research was supported by Fondecyt grant No. 1971076 to WG which is gratefully acknowledged.

\section{References}

Caldwell, J.A.R. 1997, private communication

Feast, M.W., \& Walker, A.R. 1987, ARA\&A, 25, 345

Feast, M.W., \& Catchpole, R.M. 1997, MNRAS, 286, L1

Fouqué, P., \& Gieren, W.P. 1997, A\&A, 320, 799

Gieren, W.P., Barnes, T.G., \& Moffett, T.J. 1993, ApJ, 418, 135

Gieren, W.P., Fouqué, P., \& Gómez, M. 1997, ApJ, 488, 74

Gieren, W.P., Fouqué, P., \& Gómez, M. 1998, ApJ, 496, 17

Gieren, W.P., Fouqué, P., \& Storm, J. 1999 (in preparation)

Jacoby, G.H., et al. 1992, PASP, 104, 599

Kennicutt, R.C., et al. 1998, ApJ, 498, 181

Laney, C.D., \& Stobie, R.S. 1995, MNRAS, 274, 337

Luck, R.E., Moffett, T.J., Barnes, T.G., \& Gieren, W.P. 1998, AJ, 115, 605

Madore, B.F., \& Freedman, W.L. 1991, PASP, 103, 933

Mermilliod, J.C., Turon, C., Robichon, N., Arenou, F., \& Lebreton, Y. 1997, in HIPPARCOS Venice '97, ESA SP-402, 643

Moffett, T.J., Gieren, W.P., Barnes, T.G., \& Gómez, M. 1998, ApJS, 117, 135

Oudmaijer, R.D., Groenewegen, A.T., \& Schrijver, H. 1998, MNRAS, 294, L41 
Robichon, N., Arenou, F., Turon, C., Mermilliod, J.C., \& Lebreton, Y. 1997, in HIPPARCOS Venice '97, ESA SP-402, 567

Sasselov, D., et al. 1997, A\&A, 324, 471

Szabados, L. 1997, in HIPPARCOS Venice '97, ESA SP-402, 657

Tanvir, N.R. 1997, in STScI Symp. Ser. 10, The Extragalactic Distance Scale, M. Livio, M. Donahue \& N. Panagia, Cambridge: Cambridge Univ. Press, 91

Thompson, R.J. 1975, MNRAS, 172, 455

van Leeuwen, F., \& Hansen Ruiz, C.S. 1997, in HIPPARCOS Venice '97, ESA SP-402, 689

Welch, D.L., Mateo, M., \& Olszewski, E. 1993, in IAU Coll. 139, New Perspectives on Stellar Pulsation and Pulsating Variable Stars, Cambridge: Cambridge Univ. Press, 359

\section{Discussion}

David Graff: How soon will we see results on the distances to Cepheids in LMC clusters and depth and tilt of the LMC Bar?

Gieren: Distance results for Cepheids in NGC 1866 will be available in a few months (end of 1998); results for Cepheids in other LMC clusters should emerge over the next two years, with corresponding conculsions about the depth of the LMC and tilt of the LMC bar.

John Gallagher: Have you been able to take advantage of eclipsing binaries in the MC's to provide an independant check on the angular size scales and surface brightnesses? Does this appear to be a feasible approach in the future?

Gieren: This will be an important check on the angular diameters obtained from the infrared surface brightness technique once appropriate data are available. The MACHO collaboration has turned up a Cepheid in an eclipsing binary system which will yield such a check.

Douglas Welch: One hidden assumption is that the surface brightness calibration for stable stars is the same as that for Cepheids. There is some indirect evidence that the atmospheric structure is different for Cepheids than for stable stars.

Gieren: We see in the data that the slope of the infrared surface brightness colour relation for Cepheids and stable giants and supergiants of similar colours are the same (within very small errors). This gives us much confidence that Cepheids, in spite of their pulsating atmospheres, do indeed follow the same surface brightness-colour relations as stable yellow giants and supergiants.

Nino Panagia: I guess that some of your individual errors are larger than the total error simply because you quote first the errors on individual parameters and then you give the total error on the distance determination. 